\title{
Andreas Gelhard, Ruben Hackler, Sandro Zanetti, Epistemische Tugenden. Zur Geschichte und Gegenwart eines Konzepts.
}

\section{Claire Gantet}

\section{OpenEdition}

1 Journals

\section{Electronic version}

URL: https://journals.openedition.org/ifha/11594

DOI: 10.4000/ifha.11594

ISSN: 2198-8943

\section{Publisher}

IFRA - Institut franco-allemand (sciences historiques et sociales)

\section{Electronic reference}

Claire Gantet, "Andreas Gelhard, Ruben Hackler, Sandro Zanetti, Epistemische Tugenden. Zur Geschichte und Gegenwart eines Konzepts.", Revue de I'IFHA [Online], Date of review, Online since 04 January 2022, connection on 04 August 2022. URL: http://journals.openedition.org/ifha/11594 ; DOI: https://doi.org/ 10.4000/ifha. 11594

This text was automatically generated on 4 August 2022.

All rights reserved 


\section{Andreas Gelhard, Ruben Hackler, Sandro Zanetti, Epistemische Tugenden. Zur Geschichte und Gegenwart eines Konzepts.}

Claire Gantet

\section{REFERENCES}

Andreas Gelhard, Ruben Hackler, Sandro Zanetti, Epistemische Tugenden. Zur Geschichte und Gegenwart eines Konzepts, Tübingen : Mohr Siebeck, 2019, 313 S., ISBN 978-3-16-154072-1 
Édité par un professeur de pédagogie, un doctorant en histoire des savoirs et un professeur de littérature comparée, et issu d'une journée d'étude organisée en commun par le Centre d'histoire des savoirs de l'École polytechnique de Zurich, l'Université de Zurich et le Forum de recherche interdisciplinaire de l'Université de Darmstadt, ce recueil sonde les "vertus épistémiques" à l'œuvre dans toute quête de savoirs : sans une compréhension tacite de notions telles que la patience, l'attention, la précision, l'intuition, le doute, la vraisemblance, la curiosité, la rigueur ou la fiabilité, toute production, transmission ou acquisition de savoir ne serait pas possible. Les acceptions de ces notions étant très différentes selon les contextes

\section{Epistemische} Tugenden Zur Geschichte und Gegenwart
eines Konzepts

Herausgegeben von Andreas Gelhard, Ruben Hackler und Sandro Zanetti

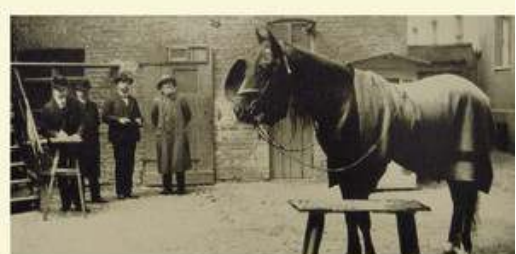

Historische Wissensforschung 11

Mohr Siebeck temporels, spatiaux et socioculturels, elles requièrent une contextualisation fine. Tout en relevant de la pratique, elles ont une valeur normative et se doublent de leurs négatifs, les "vices épistémiques». Ce recueil se veut une contribution à une épistémologie historique située dans des pratiques, et à une histoire culturelle des savoirs. Il s'attache à la conceptualisation de ces « vertus » ou de ces « vices » (I), livre quelques études empiriques (II) et dégage des perspectives de recherche (III).

Un fil rouge de l'ouvrage consiste en la distinction 1) de l'approche philosophique du sujet - la quête normative de la justification d'un savoir, c'est-à-dire d'une expression ou situation cognitive qui se veut vraie (le savoir comme « conviction vraie » selon John Greco et Ernest Sosa) - et 2) de la perspective historique empirique - l'enquête sur les pratiques liées à la gestation et la diffusion de savoirs qui ne sont pas forcément tenus pour vrais. Des contributeurs tentent de concilier les approches (ainsi Oliver Nevergelt sur Herman Paul et Miranda Fricker) ou s'affirment nettement dans l'un ou l'autre camp. L'un des protagonistes de l'approche historique est assurément Martin Mulsow, qui reconstitue les objets et pratiques, notamment figuratives, et les vertus ou vices (jusqu'au reproche de contrefaçon), du numismate Andreas Morell attaché au service du comte Anton Günther II de Schwarzburg-Sondershausen.

D'autres contributeurs sondent l'histoire d'une vertu épistémologique particulière. Ainsi, dans sa brillante étude de 1998 ici réimprimée, Michael Hagner montre comment l'attention est redéfinie de la psychologie des affects ou passions de l'âme contemporaine de Descartes à la pensée sensualiste de Condillac, tandis qu'elle devient une vertu bourgeoise, puis à la psychophysique après 1850. Marcel Lepper souligne le renouveau d'une revendication de l'honnêteté philologique, qu'il lie au programme de disciplinarisation allemand ou prussien du XIX ${ }^{e}$ siècle. L'article de Lorraine Daston sur le couple instable objectivité-impartialité, paru en anglais en 2014, est présenté en version allemande. Au XVIII ${ }^{e}$ siècle, dans le cadre de l'histoire pensée comme une maîtresse de vie enseignant la faculté de jugement comme le caractère personnel, on 
recherche une impartialité dans la quête de principe universels, qui ne revient en rien à une neutralité de valeur : il s'agit plutôt de savoir varier sa position, s'approprier la perspective d'autrui. La controverse autour de 1870 par Fustel de Coulanges et Theodor Mommsen sur la nationalité des habitants de l'Alsace-Lorraine enflamme le débat sur l'impartialité de l'historien, par la suite rejetée au profit d'une objectivité misée sur la critique des sources et sur le sacrifice de soi à la communauté. Markus Krajewski complète l'analyse par une stimulante étude de la précision et de ses médias, notamment d'après l'appareil de fiches de correction de l'ingénieur autodidacte Franz Maria Feldhaus au début du $\mathrm{XX}^{\mathrm{e}}$ siècle. Sandro Zanetti, quant à lui, sonde la valeur attachée aux sources et à leur critique. En complément, Martin Doll s'attache aux faussaires scientifiques. Au début du XVIII siècle, ils s'inscrivent dans une culture savante prompte à critiquer la pédanterie - le faussaire est autant celui qui fabrique de toutes pièces des pseudo-fossiles que le savant qui s'y laisse prendre -, ce qui change lorsque le savant va lui-même opérer des fouilles validées par un procédé expérimental.

D'autres chapitres du recueil s'attachent à une pratique et reconstituent les vertus épistémiques qu'elles impliquent. Katja Sabisch analyse ainsi les expérimentations médicales sur les êtres humains entre 1750 et 1840, autrement dit le passage de l'intégrité et de la considération intellectuelles à la neutralité entre l'expérimentateur et l'expérimenté. Plus riche est l'étude des vertus recherchées par les juges du Kaiserreich et de la République de Weimar par Ruben Hackler : ardeur au travail, quête d'aveux sans contrainte, interrogation perspicace des témoins, crainte du propre mensonge. Une contribution se plonge dans l'univers d'un auteur, celle de Cornelia Zumbusch sur l'abnégation et l'apathie chez Goethe. Une contribution finale thématise le non-savoir, l'« agnotologie » (Robert N. Proctor).

S'il n'apporte pas toujours des éléments nouveaux, le recueil est assurément stimulant, et ouvre sur toutes sortes de prolongements passionnants. On n'émettra qu'un seul regret. Même s'il est d'usage, dans les recueils collectifs allemands, de ne pas placer de conclusion en fin de volume, on aurait souhaité quelques mots de synthèse ou de pistes de recherche. On ne peut en effet qu'être frappé par la redondance des enjeux politiques dans la définition des vertus et des vices épistémiques : ils auraient pu être précisés et différenciés. Un volume au total brillant.

\section{INDEX}

Subjects: Histoire des sciences, Histoire des idées, Historiographie/Methodologie

Chronological index: Ouvrages transpériodiques 
AUTHOR

CLAIRE GANTET

Université de Fribourg (Suisse)/Universität Freiburg (Schweiz) 